\title{
Lipid Nanoparticle Topical and Transdermal Delivery: A Review on Production, Penetration Mechanism to Skin
}

\author{
Dilip Kumar Patel*, Roohi Kesharwani, Vikas Kumar \\ Department of Pharmaceutical Sciences, Sam Higginbottom University of Agriculture, Technology and Sciences, Naini, Prayagraj, Uttar Pradesh, INDIA.
}

\begin{abstract}
Nanoparticulate drug delivery systems have an advanced and modern approach over the traditional delivery system. This systematic review on nanoparticulate drug delivery containing lipid and focus on preparation, challenges and advancement of delivery of the drug via topical and transdermal route. The first era of lipid nanoparticles were SLN and have more sustaining action as well as suitable for the higher permeation of drug. The NLC is the second generation lipid nanoparticles developed to overcome the limitation associated with SLN i.e low drug loading capacity, polymorphism of solid lipid. Production of solid lipid nanoparticles and nanostructured lipid carriers are produced by a variety of technologies which explored in the current review. Lipid nanoparticle has various properties for topical use of cosmetics and other pharmaceutical formulation, i.e. controlled and sustained release of medicaments, Physical and chemical stability of active pharmaceutical ingredients, targeted release, film formation and enhancing penetration with the enhancement of skin hydration. Skin hydration plays a major role in the topical delivery of API as it hydrates the skin which leads to opening of pores of skin. Due to occlusion nature of lipid nanopar-
\end{abstract}

\section{INTRODUCTION}

Lipid particles which are made up of triglycerides, waxes or unsaturated fats as lattice lipids are in effect seriously explored as potential transporter frameworks, specifically for lipophilic substances. ${ }^{1}$ These nano range particles were created in the most recent decade of the most recent century as option delivery system to another conventional approach. Solid Lipid Nanoparticles (SLN) and Nanostructured Lipid Carrier (NLC) are the two fundamental sorts of a nanoparticulate systems containing lipid. Because of the lipid framework, the little molecule estimate and related adhesive characters, the lattice arrangement time of this particulate system on the skin is drawn out. ${ }^{2}$ Within the past few decades, nanotechnology, in particular, manufacturing of nanoparticles have found unprecedented attention in broad areas of science. A PubMed search ("nanoparticles") reveals, in past year alone (2016), there were"19,338" articles published related to various aspects of nanoparticle technology. ${ }^{3-5}$

\section{ADVANTAGES OF LIPID NANOPARTICLE OVER CONVENTIONAL PARTICULATE CARRIERS}

- $\quad$ Site-specific drug targeting ${ }^{6}$ is possible due to its narrow size distribution.

- It can be formulated for Controlled and Sustained delivery of API.

- The encapsulated medication is shielded from the biochemical degradation.

- High loading efficiency

- The incorporation of lipophilic and hydrophilic drugs is possible.

- The lyophilization and spray drying technique utilizes to achieve higher stability. ticles trans epidermal water loss decline which softening the skin. The use of biodegradable grade lipid makes it more suitable because it does not cause any toxicity as created by polymeric nanoformulations. Furthermore, a discussion about the benefit/risk ratio of a nanoparticulate system containing lipids also explored in this paper. The SLN and NLC are a "nanosafe" carrier for the delivery of active pharmaceutical via topical route.

Key words: Lipid nanoparticles, SLN, NLC, Topical Delivery, Skin Permeation.

Correspondence

Mr. Dilip Kumar Patel,

Department of Pharmaceutical Sciences, Sam Higginbottom University of Agriculture, Technology and Sciences, Naini, Prayagraj-211007, Uttar Pradesh, INDIA.

Phone no: +91 9936764324

Email: dilippatel87mph@gmail.com

DOI: 10.5330/ijpi.2019.4.28

- It produces stable formulation.

- Surface alteration can be effortlessly performed.

\section{DEFINITION OF SOLID LIPID NANOPARTICLE (SLN)}

It is presented in 1991 which provides an advanced transporter framework to the conventional colloidal system, for example, emulsions, liposomes and polymeric small scale and nanoparticles. ${ }^{7}$ They are submicron colloidal carrier extending from 40 to $1000 \mathrm{~nm} .{ }^{8}$ It offers novel characters, for example, little size, large medication loading and their capability to enhance the activity of API.

These are particles produced using solid and liquid lipid mixes delivered by homogenization with utilizing higher pressure. Particles beneath $80 \mathrm{~nm}$ are more hard to create on the grounds that regularly they don't recrystallize, The SLN are dispersed in a watery external stage and balanced out with the use of surfactants, e.g., Tween 80 , lecithin, etc. Then again, they can be created surfactant-free utilizing steric stabilizers (e.g. poloxamer 180) or an external of expanded consistency (e.g. ethylcellulose solution). SLN can likewise be created in nonaqueous media, e.g., PEG-600 or oils like Miglyol 812. Generation in lower grade polyethylene glycol gives a formulation which can be specifically utilized in soft gelatin capsules. ${ }^{9,10}$

\section{TYPES OF SLN}

The kind of SLNs is depended upon the nature of the incorporated drug and lipid, the solubility of the incorporated drug in the softened lipid (Figure 1), kind of preparation and the preparation temperature. ${ }^{11}$ 


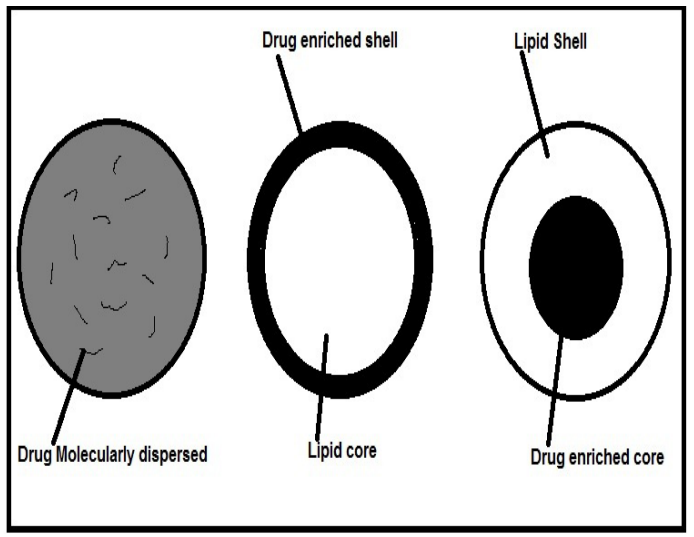

Figure 1: Various forms of SLN enriched with medicament.

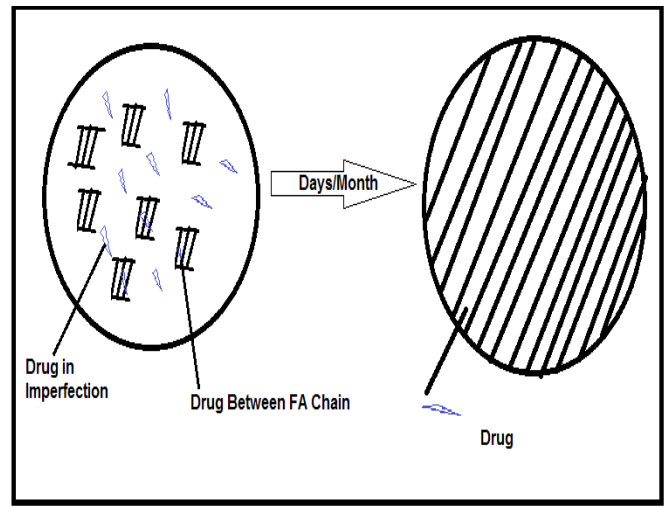

Figure 2: The expulsion of medicament during storage of SLN dispersions, ${ }^{4}$ converted to orderly arranged lipid crystal.

1. SLN, Type I or homogenous matrix Type

2. SLN, Type II or API enriched shell Type

3. SLN, Type III or API enriched core Type

\section{MAJOR DRAWBACK RELATED TO SLN AND ITS PRODUCTION TECHNIQUE}

The review by Mehnert highlights these aspects. ${ }^{2,12}$

- The drug loading capacity is low.

- $\quad$ Drug expel out during after certain periods (Figure 2)

- Greater Aqueous content of SLN dispersions leads to instability.

The Nanostructured lipid carrier has been produced to defeat the downsides related to the above nanoparticulate system. They are thought to be the $2^{\text {nd }}$ era of lipid nanoparticulate systems. Comparison with SLN, it demonstrates a high stacking limit with respect to active pharmaceutical ingredients products by making a low ordered arranged network through the mixing of both type lipid, a higher API stacking can be accomplished. Along these lines, the NLC has an expanded API stacking capacity in contrast with solid lipid nanoparticle likelihood of medication expulsion is less. ${ }^{13,14}$

\section{THE NOVEL CONCEPT OF NLC}

There are three kinds of NLC can be summarized

1. The imperfect type of NLC

2. The amorphous type of NLC

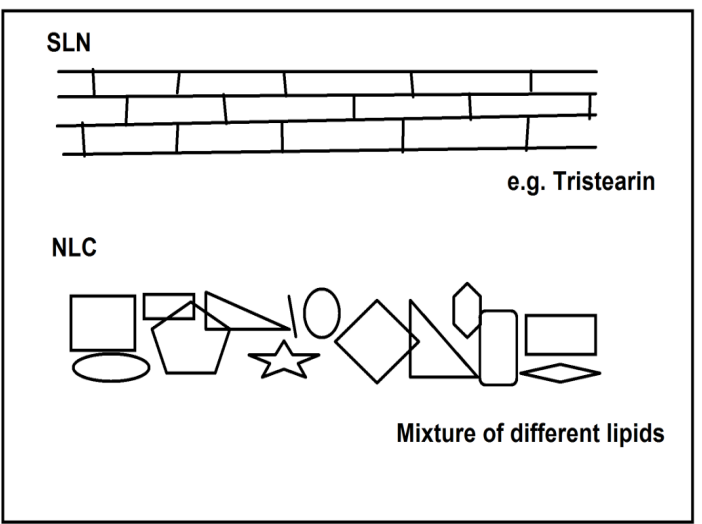

Figure 3: Lattice order in SLN resembles a brick wall (upper) and structure with disordered due to spacially very different molecules in NLC type.

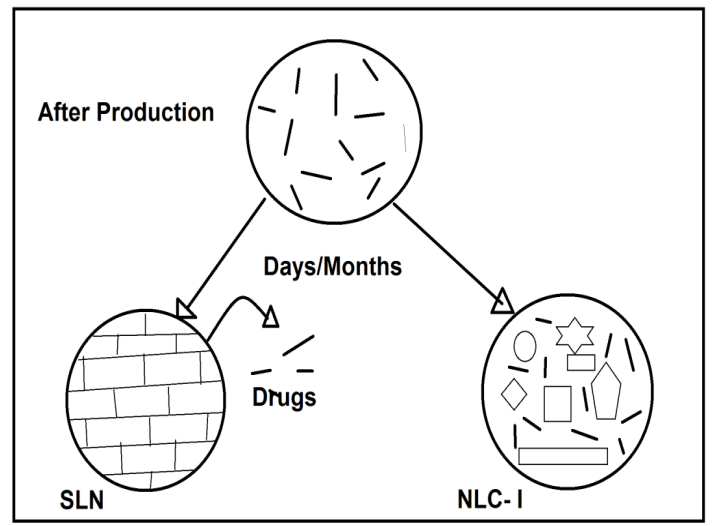

Figure 4: The process of Crystallization in SLN (perfect) \& NLC I with imperfection.

3. The multiple types of NLC

The major issue in SLN is the formation of ordered structure. Utilizing different molecules to assemble the lattice creates imperfection to provide space for the active pharmaceutical ingredients (Figure 3 and 4). Expulsion of the drug is brought about by a continuous crystallization process towards an ordered structure. In this manner, by staying away from crystallization, one can maintain a strategic distance from these obstructions which is acknowledged in the NLC type 2. The mixture of lipid is solid is in an amorphous form yet it is not crystallized (Figure 5). This can be accomplished by blending unique lipids. ${ }^{15}$ The third kind of NLC is multiple types, it is practically identical to multiple emulsion. This NLC type utilizes the way that for various medications, the solubility shows in oils is more than solid type ${ }^{16}$ (Figure 6).

\section{PRODUCTION TECHNIQUES FOR LIPID NANOPARTICLES}

Lipid Nanoparticle are prepared from solid lipid, Liquid lipid, emulsifying agent and water/ solvent by utilizing various technologies ${ }^{2,17-30}$ and are discussed below

1. High-pressure homogenization Technique:

a. Hot homogenization

b. Cold homogenization

2. Ultrasonication/high-speed homogenization Technique

3. Solvent Emulsification Evaporation Technique

4. Solvent emulsification-diffusion Technique 


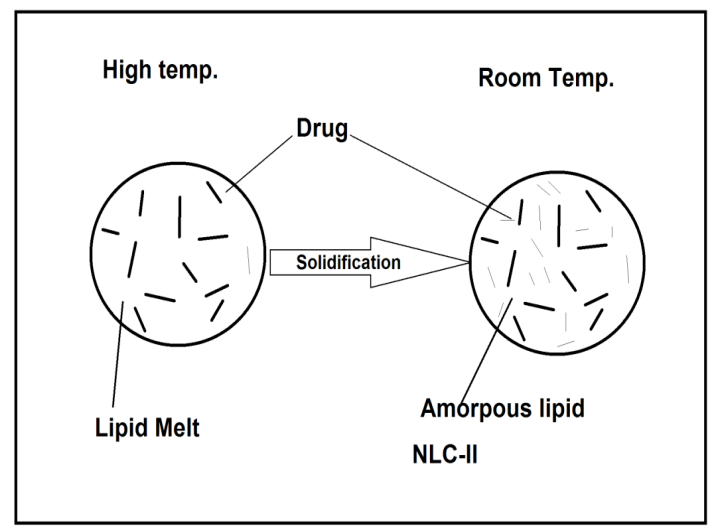

Figure 5: In Type II NLC the solidification of lipid in the solid amorphous phase.

\section{Supercritical fluid Technique'}

6. Microemulsion based Technique

7. Spray drying Technique

8. Double emulsion Technique

9. Precipitation technique

10. Solvent injection technique

11. Membrane contractor technique

12. Film-ultrasound dispersion

\section{CHARACTERIZATION OF LIPID NANOPARTICLE}

There are various parameters to characterize the nanoparticulate system which included measurement of particle size, zeta potential, crystallinity and morphology, etc. ${ }^{31}$

\section{Particle Size and Zeta Potential}

There are various methods for particle size measurement like transmission electron microscopy, Scanning electron microscopy, scanning tunneling microscopy, atomic force microscopy, Photon Correlation Spectroscopy (PCS), Freeze-fracture Electron Microscopy (FFEM). Coulter counter is infrequently used to gauge molecule estimate due to troubles in the evaluation of small nanoparticle and the need for electrolytes which may destabilize colloidal scatterings. ${ }^{29}$ Zeta potential estimation can be completed utilizing zeta meter. The estimation is done by diluting nanodispersion to 50 times with the pre dispersion preparation medium. A higher estimation of zeta potential may prompt deaggregation of particles without other entangling components. Zeta potential estimations permit forecasts about the stability of dispersions during storage. It is observed that the requirement for full electrostatic adjustment is $60 \mathrm{mV} \cdot{ }^{17}$

\section{Static light scattering technique}

It is also named as Fraunhofer diffraction method, this technique is quick and tough, but it requires more cleanliness than Dynamic light scattering technique and propel information of the particles optical qualities, Static Light Scrambling (SLS) is an outfit strategy in which the example of light scattered from an answer of particles is gathered and fit to central electromagnetic conditions in which size is the essential variable. ${ }^{17}$

\section{Dynamic Light Scattering (DLS)}

This technique is well known as Photon correlation spectroscopy which records the variety in the power of scattered light on the $\mu$ sec. time scale. This variety comes about because of the impedance of light scattered through each particle affected by Brownian movement and is measured by the accumulation of an autocorrelation work. This capacity is fit to

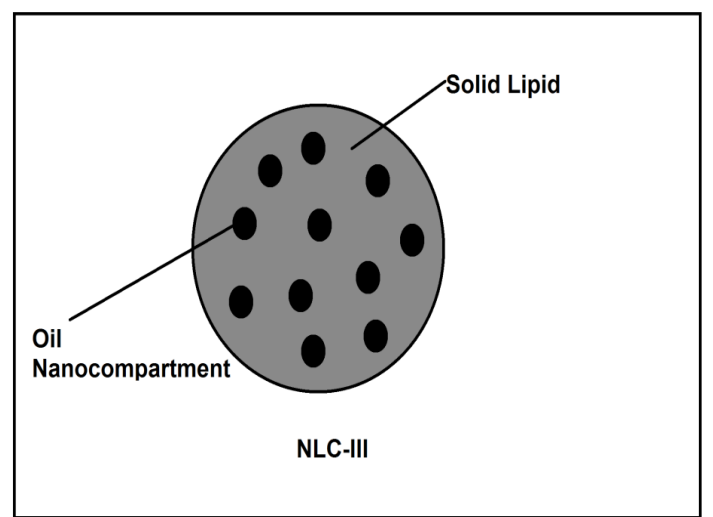

Figure 6: Multiple type NLC.

an exponential, or some blend or change thereof, with the comparing rate constant(s) being identified with the dispersion coefficient(s). Using standard suppositions of round size, low fixation and known thickness of the suspending medium, molecule size is computed from this coefficient. The benefits of the technique are the speed of investigation, absence of needed alignment and affectability to submicron-sized particles. ${ }^{29}$

\section{Electron Microscopy}

There are various electron microscopy such as Scanning Electron Microscopy (SEM) technique is based on electron scanning principle and it provides all available information about the nanoparticles at the nanoscale level. A wide range of reviews is available, where people used this technique to study not only the morphology of their nanomaterials but also the dispersion of nanoparticles in the bulk. Transmission Electron Microscopy (TEM) gives an approach to specifically watch nanoparticles and a physical picture of nanoparticles with the previous strategy being better for determining morphology. TEM has a littler size breaking point of location, is a decent approval for different techniques and one must be conscious of the measurably little specimen estimate and the impact that vacuum can have on the particles..$^{10,31,32}$

\section{Acoustic methods}

This technique utilizes to measures the constriction of sound waves as a method for deciding size through the fitting of physically significant conditions. What's more, the swaying electric field produced by the development of charged particles affected by acoustic vitality can be identified to give data on charge observed on the surface..$^{23}$

\section{X-ray diffraction or Differential Scanning Calorimetry (DSC)}

Both are done for the assurance of the level of crystallinity of the molecule scattering. The rate of crystallinity utilizing DSC is assessed by correlation of the liquefying enthalpy per gm of the mass substance with the softening enthalpy per gm of the scattering. ${ }^{2,17}$

\section{Atomic Force Microscopy AFM}

In this procedure, a probe tip with atomic-scale sharpness is a framework over a specimen to create a topological guide in view of the strengths at play between the surface and the tip. The test can be dragged over the example (contact mode), or permitted to drift simply above (noncontact mode), with the correct way of the specific drive utilized serving to recognize among the other related techniques. This determination is realistic with this approach, which alongside the capacity to outline test as indicated by properties notwithstanding size. ${ }^{2,23}$ 


\section{LIPID NANOPARTICLE AS TOPICAL DRUG DELIVERY SYSTEMS}

This type of medication application has been acquainted for a long time with accomplishing a few purposes on various levels (skin surface, epidermis, dermis and subcutaneous layer). In any case, a few issues have been accounted for with the ordinary topical formulation e.g. low uptake because of the barrier work of the stratum corneum and movement of API to the systemic flow. Medicament application to the skin surface maintains a strategic distance from the real changes of plasma levels normal for the rehashed organization of quickly wiped out medications while it additionally permits dodging the primary entry of medicament via liver after intestinal ingestion. Therefore transdermal medication application has increased as yet expanding significance for systemic treatment e.g. with medications subject to broad first-pass disposal. ${ }^{33}$ The small size guarantees a nearby contact to the horny layer and can expand the measure of medication infiltrated into the skin. ${ }^{34,35}$

\section{SKIN MORPHOLOGY FOR TOPICAL AND TRANSDERMAL DELIVERY}

API in contact with the skin surface can infiltrate by either three potential pathways: through the sweat channels, by means of the hair follicles and sebaceous organs (by and large called the shunt or appendageal course), or straightforwardly over the stratum corneum. The relative significance of the shunt or appendageal course versus transport over the stratum corneum has been wrangled by researchers throughout the years and is additionally confounded by the absence of an appropriate exploratory model to allow division of the three pathways. In vitro explores to have a tendency to include the utilization of hydrated skin or epidermal films so members are shut by the swelling related to hydration. Scheuplein and associates suggested that a follicular shunt course was in charge of the pre enduring state penetration of polar atoms and flux of vast polar particles or particles that experience issues diffusing over the in-place stratum corneum. ${ }^{36}$ The three fundamental elements deciding the transdermal penetration of medications are the versatility of medication in the vehicle, the arrival of medication from the vehicle and the permeation of medication into the skin. These variables influence either the thermodynamic movement that drives the medication into the skin or the penetrability of medication in the skin, especially stratum corneum. Lipid nanoparticle-based gel enhances the transdermal conveyance of a few medications over the regular topical arrangements, for example, emulsions and gels. Among the physical properties that make lipid-based gel appealing as transdermal medication conveyance vehicles are their straightforward nature, which implies that the item is stylishly satisfying, as well as permits simple representation of any tainting. Altogether the little bead measure gives a huge interfacial region to fast medication release, thus the medication ought to display an improved bioavailability, empowering a decrease in dosage, more reliable fleeting profiles of medication assimilation and the security of medications from the antagonistic condition of the body. Since lipid nanoparticle contains surfactants (co-surfactants) and different excipients, they may serve to expand membrane permeation of API by:

- Disrupting the intercellular two-layer lipid structure

- Interacting with the intracellular proteins of the horny layer

- Improving the parceling of medication into the horny layer. ${ }^{37}$

\section{MECHANISM OF DRUG PENETRATION}

The particles have sufficient solvency in water and oil, with high oil/water diffusion coefficients. A molecular weight under $0.6 \mathrm{kDa}$ may infiltrate the skin. Along these lines, the topical organization is restricted to hydrophobic and low molecular weight drugs. Since most medications are hydrophilic, have low oil/water partition coefficients and ionic characters. They don't effectively cross the stratum corneum. The bioavailability of medications pervading the skin can be expanded by utilizing nanoparticulate containing lipid which obtained as small particulate size having close contact with stratum corneum. Nanosized particles can reach shallow intersections of SC and wrinkles between corneocytes islands, permitting shallow spreading of the API. Taking after the vanishing of water from the nanoparticulate systems subsequent to applying the surface of the skin, particles frame a cement layer blocking the skin. Hydration of stratum corneum along these lines upgrades to lessen corneocyte pressing and width bury corneocytes crevices and furthermore impacts apportioning of the medication into stratum corneum. Over the $100 \mathrm{~nm}$, the molecule size of lipid nanoparticle is not considered to saturate the stratum corneum due to their measurements and unbending nature. Lipid nanoparticles can possibly convey drugs by means of the follicles. Moreover, every follicle is related to sebaceous organs, which discharge the sebum, making a domain improved in lipids. This condition is helpful for catching of lipid nanoparticles. Sebum is a blend of triglycerides, squalene and waxes. Some glyceride lipids introduce in lipid nanoparticle may quicken the passageway into the follicles/sebaceous organs. $^{38}$

\section{TOPICAL BENEFIT OF LIPID NANOPARTICLE}

\section{Skin Hydration and Elasticity}

The diminishment of Transepidermal Water Loss (TEWL) brought about by occlusion prompts an expansion in skin hydration after dermal utilization of lipid nanoparticulate drug delivery. An in vivo thinks about demonstrated that the SLN-containing o/w cream expanded the skin hydration fundamentally more than the customary o/w cream. A noteworthy higher increment in skin hydration was observed by Muller et al. for Lipid nanoparticles containing cream contrasted with traditional cream which was explored for 28 days..$^{39-41}$

\section{Skin Permeation and Drug Targeting}

The horny layer in the skin has regularly a water percentage of $20 \%$ and gives generally a powerful obstruction against percutaneous retention of exogenous substances. Skin hydration subsequent to applying SLN or NLC prompts a lessening of corneocytes pressing and an expansion in the span of the corneocyte's crevices. This will encourage the percutaneous ingestion and medication infiltration to the more profound layers of the skin. ${ }^{33}$ Ricci et al. in his research explored the in vitro infiltration of indomethacin NLC based gel and gel simple gel through the horny layer and epidermis. He likewise researched the release of indomethacin in vivo study by tape-stripping test and the anti-inflammatory movement utilizing the UV-B by observing erythema. In this work, it was observed that the indomethacin-stacked NLC gel shows higher anti-inflammatory impact taking. The permeation through the horny layer and epidermis in vitro from indomethacin-stacked NLC gel was not exactly from a gel without NLC. ${ }^{42}$

\section{Skin Occlusion}

The lipid film development on the upper layer of the skin and the ensuing impediment impact was accounted for. By utilizing small lipid particles, which are created from profoundly crystalline and low softening point lipids, the most noteworthy impediment will become to. Souto et al. found a higher occlusive figure for SLN correlation with NLC of similar lipid content. Contrasting NLC and diverse oil content demonstrated that an expansion in oil content prompts a reduction of the occlusive component. ${ }^{40,43}$ 


\section{Administrative Status of Excipients}

One obstacle for a definition to be acquainted with the market is the utilization of excipients having no acknowledged status. For topical lipid nanoparticle, all excipients utilized as a part of current topical restorative and dermal pharmaceutical items can be utilized. What's more, GRAS substances and substances with acknowledged GRAS status can be utilized (Code of Federal Regulations, Food and Drugs 21). ${ }^{34}$

\section{Skin Benefit/Risk Ratio}

Skin decay and systemic toxic effect happened in the wake of applying ordinary prednicarbate cream could be maintained a strategic distance from when this medication was figured as lipid nanoparticle. Predinicarbate take-up was improved and it was collected in the epidermis with a low quantity in the dermis. ${ }^{44}$ One of the real disservices related to the topical use of tretinoin is the local skin disturbance, for example, erythema, peeling and consuming and additionally expanded affectability to daylight. In the in vitro saturation concentrates through rodent skin they found that SLN based tretinoin gel has a pervasion profile tantamount to that of the market tretinoin cream. Yet, then again, Draize fix test demonstrated that SLN based tretinoin gel brought about amazingly less erythremic scenes contrasted with the at present promoted tretinoin cream and thus, a superior advantage/hazard proportion is normal for the details containing tretinoin-stacked SLN. ${ }^{45}$

\section{CONCLUSION}

Lipid nanoparticles are the novel carrier system for the topical and transdermal delivery systems. The generation of this bearer system is plausible in the research center and on a substantial scale. The elements of a nanoparticulate system containing lipids that are profitable for topical utilization of pharmaceutical items have been accounted for, e.g. occlusive properties, modified release, the increment of skin permeation related to a targeting effect, increment in skin hydration and evasion of systemic take-up. Different medications that topical details containing lipid nanoparticles can an increased activity and in addition delayed/ prolonged action was accounted for whiles the benefits/risk proportion was expanded for some medications and improve the infiltration into the skin expanding treatment proficiency focus on the epidermis. Besides, decrease systemic toxic and side effects announced by different written works.

\section{ACKNOWLEDGEMENT}

None.

\section{CONFLICT OF INTEREST}

The authors declare that there are no conflicts of interest.

\section{ABBREVIATIONS}

SLN: Solid Lipid Nanoparticles; NLC: Nanostructure Lipid Carrier; DSC: Differential Scanning calorimetry; SEM: Scanning Electron Microscopy; PCS: Photon Correlation spectroscopy; AFM: Atomic Force Microscopy.

\section{REFERENCES}

1. Bunjes $H$, Koch MHJ. Saturated phospholipids promote crystallization but slow down polymorphic transitions in triglyceride nanoparticles. J Control Release. 2005;107(2):229-43.

2. Mehnert W, Mader K. Solid lipid nanoparticles: Production, characterization and applications. Adv Drug Deliv Rev. 2001;47(2-3):165-96.

3. Muller RH, Mader K, Lippacher A, Jenning V. Solid-liquid (semi-solid) liquid particles and method of producing highly concentrated lipid particle dispersions. German Patent Application. 2000;199
4. Radtke M, Muller RH. Nanostructured lipid carriers: The new generation of lipid drug carriers. New Drugs. 2001;2:48-52.

5. Pub Med. 2017. <https://www.ncbi.nlm.nih.gov/pubmed>

6. Ekambaram PA. Sathali AH, Priyanka K. Solid Lipid Nanoparticles: A Review. Sci Revs Chem Commun. 2012;2(1):80-102

7. Westesen K. Novel lipid-based colloidal dispersions as potential drug administration systems-expectations and reality. Coll Polym Sci. 2000;278(7):608.

8. Neda N, Hadi V, Parvin ZM. Solid Lipid Nanoparticles and Nanostructured Lipid Carriers: Structure, Preparation and Application. Advanced Pharmaceutical Bulletin. 2015;5(3):305-13.

9. Muller RH, Runge SA. Solid Lipid Nanoparticles (SLN) for controlled drug delivery: Submicron Emulsions in Drug Targeting and Delivery Harwood Academic Publishers. Amsterdam. 1998;219-34

10. Ramteke KH, Joshi SA, Dhole SN. Solid Lipid Nanoparticle: A Review. IOSR Journal of Pharmacy. 2012;2(6):34-44

11. Mehnert W, Muhlen AZ, Dingler A, Weyhers H, Muller RH. Solid Lipid Nanoparticles (SLN)-einneuartiger Wirkstoff- Carrier für Kosmetika und Pharmazeutika: II. WirkstoffInkorporation, Freisetzung und Sterilizierbarkeit. Pharm Ind 1997;59:511-4.

12. Patidar $A$, Thakur DS, Kumar $P$, Verma J. A review on novel lipid-based carrier. International J of Pharmacy and Pharmaceutical Sciences. 2010;2(4):30-5.

13. Saupe A, Wissing SA, Lenk A, Schmidt C, Muller RH. Solid Lipid Nanoparticles (SLN) and Nanostructured Lipid Carriers (NLC) - Structural investigations on two different carrier systems. Bio Med Mater Eng. 2005:15(5):393-402.

14. Joshi M, Patravale V. Nanostructured lipid carrier (NLC) based gel of celecoxib. Int J Pharm. 2008;346(1-2):124-32.

15. Kaur S, Nautyal U, Singh R, Singh S, Devi A. Nanostructure Lipid Carrier (NLC): The new generation of lipid nanoparticles. Asian Pacific Journal of Health Sciences. 2015;2(2):76-93.

16. Muller RH, Radtke M, Wissing SA. Nanostructured lipid matrices for improved microencapsulation of drugs. Int J Pharm. 2002:242(1-2):121-8.

17. Rabinarayan P, Padilama S. Preparation and Characterization of Solid Lipid Nanoparticles-A Review. Current Drug Discovery Technologies. 2012;9(1):4-5

18. Lander R, MangerW, Scouloudis M, Ku A, Davis C, Lee AG. Homogenization-a mechanistic study. Biotechnol Prog. 2000;16(1):80-5

19. Jahnke S, Muller RH, Benita S, Bohm B. Emulsions and Nanosuspensions for the Formulation of Poorly Soluble Drugs. Medpharm Scientific Publishers, Stuttgart. 1998; 177-200

20. Gowda DV, Karamsetty VM. Nano structured lipid carrier-based drug delivery system. Journal of Chemical and Pharmaceutical Research. 2016;8(2):627-43.

21. DeLabouret A, Thioune O, Fessi H, Devissaguet JP, Puisieux F. Application of an original process for obtaining colloidal dispersions of some coating polymers preparation, characterization, industrial scaling up. Drug Dev Ind Pharm. 1995:21(2):229-41.

22. Siekmann B, Westesen K. Investigations on solid lipid nanoparticles prepared by precipitation in o/w emulsions. Eur J Pharm Biopharm. 1996;43:104-9.

23. Garud A, Singh D, Garud N. Solid Lipid Nanoparticles (SLN): Method, Characterization and Applications. International Current Pharmaceutical Journal. 2012:1(11):384-93.

24. Khan S. Solid Lipid Nanoparticles: A Review. World Journal of Pharmacy and Pharmaceutical Sciences. 2012:1(1):96-115.

25. Gasco MR. Method for producing solid lipid microspheres having a narrow size distribution. The United States Patent. 1993:5:236-50.

26. Moulik SP, Paul BK. Structure, dynamics and transport properties of microemulsions. Adv Coll Interf Sci. 1998;78(2):99-195.

27. Lee J, LeeY, Kim J, Yoon M, Choi WY. ss Arch Pharm Res. 2005;28(9):1097-102

28. Kamble MS, Vaidya KK, Bhosale AV. Solid Lipid Nanoparticles And Nanostructured Lipid Carriers-An Overview. IJPCBS. 2012;2(4):686.

29. Mukheriee S, Ray S, Thakur RS. Solid lipid nanoparticles (SLN): A Modern Formulation Approach in Drug Delivery System. Indian Journal of Pharmaceutical Sciences. 2009;71(4):349-58.

30. Schubert MA, Muller RH, Goymann CC. Solvent injection as a new approach for manufacturing lipid nanoparticles-evaluation of the method and process parameters. EJPB. 2003;55(1):125.

31. Syed AAR, Ayman MS. Applications of Nanoparticle Systems in Drug Delivery Technology. Saudi Pharmaceutical Journal. 2018;26(1):64-70.

32. Khan SK. Preparation and characterization of single-walled carbon nanotube/ nylon 6,6 nanocomposites. Instrum Sci Technol. 2016;44(4):435-44

33. Schafer-Korting M, MehnertW, Korting HC. Lipid nanoparticles for improved topical application of drugs for skin diseases. Adv Drug Deliv Rev. 2007;59(6):42743.

34. Muller RH, Radtke M, Wissing SA. Solid Lipid Nanoparticles (SLN) and nanostructured lipid carriers (NLC) in cosmetic and dermatological preparations. Adv Drug Deliv Rev. 2002;54(1):131-55.

35. Zur MA, Mehnert W. Drug release and release mechanism of prednisolone loaded solid lipid nanoparticles. Pharmazie. 1998;53:552-5.

36. Barry BW, Woodford R. Comparative bio-availability of proprietary topical corti- 
costeroid preparations: Vasoconstrictor assays on thirty creams and gels. $\mathrm{Br} \mathrm{J}$ Dermatol. 1974;91(3):323-38.

37. Benson HAE. Transdermal Drug Delivery: Penetration Enhancement Techniques. Current Drug Delivery. 2005;2(1):23-33.

38. Baroli B. Penetration of nanoparticles and nanomaterials in the skin: Fiction or reality?. J Pharm Sci. 2009;99(1):21-50.

39. Chia-Lang F, Al-Suwayeh S, Jia-You F. Nanostructured lipid carriers (NLCs) for drug delivery and targeting. Recent Patent on Nanotechnology. 2013;7(1):41-55.

40. Wissing SA, Muller RH. The influence of solid lipid nanoparticles on skin hydration and viscoelasticity-in vivo study. Eur J Pharm Biopharm. 2003;56(1):67-72.

41. Muller RH, Petersen RD, Hommoss A, Pardeike J. Nanostructured lipid carriers
(NLC) in cosmetic dermal products. Adv Drug Del Reviews. 2007;59(6):522-30.

42. Ricci M, Puglia C, Bonna F, Di GC, Giovagnoli S, Rossi C. Evaluation of indomethacin percutaneous absorption from nanostructured lipid carriers (NLC): In vitro and in vivo studies. J Pharm Sci. 2005;94(5):1149-59.

43. Teeranachaideekul V, Boonme P, Souto EB, Muller RH, Junyaprasert VB. Influence of oil content on physicochemical properties and skin distribution of Nile red-loaded NLC. J Control Release. 2008;128(2):134-41.

44. Maia CS, Mehnert W, Schafer-Korting M. Solid lipid nanoparticles as drug carriers for topical glucocorticoids. Int J Pharm. 2000;196(2):165-7.

45. Shah KA, Date AA, Joshi MD, Patravale VB. Solid Lipid Nanoparticles (SLN) of tretinoin: Potential in topical delivery. Int J Pharm. 2007;345(1-2):163-71.

Cite this article: Patel DK, Kesharwani R, Kumar V. Lipid Nanoparticle Topical and Transdermal Delivery: A Review on Production, Penetration Mechanism to Skin. Int. J. Pharm. Investigation. 2020;9(4):148-53. 